\title{
Conventionally assessed voluntary activation does not represent relative voluntary torque production
}

\author{
R. D. Kooistra - C. J. de Ruiter - A. de Haan
}

Accepted: 6 February 2007/Published online: 15 March 2007

(C) Springer-Verlag 2007

\begin{abstract}
The ability to voluntarily activate a muscle is commonly assessed by some variant of the twitch interpolation technique (ITT), which assumes that the stimulated force increment decreases linearly as voluntary force increases. In the present study, subjects $(n=7)$ with exceptional ability for maximal voluntary activation (VA) of the knee extensors were used to study the relationship between superimposed and voluntary torque. This includes very high contraction intensities (90-100\%VA), which are difficult to consistently obtain in regular healthy subjects (VA of $\sim 90 \%$ ). Subjects were tested at 30,60 , and $90^{\circ}$ knee angles on two experimental days. At each angle, isometric knee extensions were performed with supramaximal superimposed nerve stimulation (triplet: three pulses at $300 \mathrm{~Hz}$ ). Surface EMG signals were obtained from rectus femoris, vastus lateralis, and medialis muscles. Maximal VA was similar and very high across knee angles: $97 \pm 2.3 \%$ (mean $\pm \mathrm{SD}$ ). At high contraction intensities, the increase in voluntary torque was far greater than would be expected based on the decrement of superimposed torque. When voluntary torque increased from $79.6 \pm 6.1$ to $100 \% \mathrm{MVC}$, superimposed torque decreased from $8.5 \pm 2.6$ to $2.8 \pm 2.3 \%$ of resting triplet. Therefore, an increase in VA of $5.7 \%$ (from $91.5 \pm 2.6$ to $97 \pm 2.3 \%$ ) coincided with a much larger increase in voluntary torque $(20.4 \pm$
\end{abstract}

R. D. Kooistra · C. J. de Ruiter · A. de Haan ( $\square)$ Institute for Fundamental and Clinical Human Movement Sciences, Vrije Universiteit, Van der Boechorststraat 9, 1081 BT Amsterdam, The Netherlands

e-mail: A.dehaan@fbw.vu.nl

\section{A. de Haan}

Institute for Biophysical and Clinical Research into Human Movement, Manchester Metropolitan University, Cheshire, UK
6.1\%MVC) and EMG $(33.9 \pm 6.6 \% \max )$. Moreover, a conventionally assessed VA of $91.5 \pm 2.6 \%$ represented a voluntary torque of only $79.6 \pm 6.1 \% \mathrm{MVC}$. In conclusion, when maximal VA is calculated to be $\sim 90 \%$ (as in regular healthy subjects), this probably represents a considerable overestimation of the subjects' ability to maximally drive their quadriceps muscles.

Keywords Voluntary activation - Neural drive . EMG $\cdot$ Muscle length $\cdot$ Twitch interpolation

\section{Introduction}

When a supramaximal electrical stimulus is applied to the nerve of a muscle during a voluntary isometric contraction, the contractile response of any motor units not yet (fully) recruited will lead to a force increment. Merton (1954) first reported a linear decrease of this superimposed force increment as voluntary contraction intensity increased in the adductor pollicis muscle. Hence, as voluntary activation (VA) increases, the force increment as a result of electrical stimulation decreases. For the most accurate assessment of maximal VA, Behm et al. (1996) recommended using the maximal voluntary contraction (MVC) with a superimposed electrical stimulus, which is now common practice (Allen et al. 1995; Babault et al. 2003; Becker and Awiszus 2001; Behm et al. 2001; de Ruiter et al. 2004; Maffiuletti and Lepers 2003; Newman et al. 2003; Oskouei et al. 2003; Suter and Herzog 1997). Maximal VA is generally quantified by expressing the 'twitch' force increment on an MVC as a percentage of resting twitch force. Subtraction from $100 \%$ results in a value for maximal VA.

Generally, high levels of VA ( $90 \%)$ for the knee extensors are achieved by regular healthy subjects (e.g. 
Babault et al. 2003; Behm et al. 2001; Bulow et al. 1993; de Ruiter et al. 2004; Newman et al. 2003; Oskouei et al. 2003; Suter and Herzog 1997). An important implicit meaning of a VA level of $90 \%$ is that $90 \%$ of the maximal torque capacity (MTC) is reached, and that at most, a $10 \%$ further increase in torque is possible. In our experience, however, a greater increase in torque can be observed than the obtained VA level suggested when subjects succeed in improving their MVC in a later attempt during the same session. This suggests that VA can be overestimated using the conventional method of superimposed stimulation. This would have important consequences for conclusions drawn regarding calculated VA. The inability of patient groups to access their muscles' potential could be strongly underestimated. This has implications for the estimations made of the effects of paralytic poliomyelitis on strength and endurance for instance (Grabljevec et al. 2005). Furthermore, many studies have been performed using target force levels expressed as \%MVC (de Ruiter et al. 2006; Grabljevec et al. 2005; Hisaeda et al. 2001; Kalmar and Cafarelli 1999; $\mathrm{Ng}$ et al. 1994; Place et al. 2005). For example, the \% MVC at which full occlusion of blood flow of the knee extensors occurs during isometric contraction (de Ruiter et al. 2006). Clearly, knowledge of the potential of a far greater torque increase than, for example, the $10 \%$ implied by a VA of $90 \%$ is very important.

In our experience, in subjects capable of consistently high levels of maximal VA ( $97 \%)$, the superimposed torque increment is already very small at high contraction intensities $(\sim 70 \% \mathrm{MVC})$. Further increases in voluntary torque show only minor reductions of the superimposed torque increment. This is in accordance with the modelling study of Herbert and Gandevia (1999); for the adductor pollicis muscle they show that large increases of motoneuronal excitation near MVC will be accompanied by only minor reductions in the size of the superimposed force. At high contraction intensities, therefore, the \%VA does not necessarily represent the same percentage of the muscles' maximal torque capacity. The first aim of the present study was therefore to assess the relationship between calculated VA and voluntary torque of the knee extensors at high contraction intensities. To achieve the best possible assessment of the relationship between conventionally assessed VA and voluntary torque (as \%MVC), in the present study, only those individuals capable of consistently high levels of maximal VA were included.

An additional concern of using the ITT is the dependence of the calculated VA on the size of the resting twitch. Following a single electrical stimulus, tendon slack must first be taken up before resting peak twitch torque is reached. Therefore, during an isometric contraction, where most of the tendon slack has already been taken up, the twitch torque increment will be relatively large compared to the twitch torque at rest (Belanger and McComas 1981). By scaling the superimposed twitch to resting twitch torque, calculated VA will thus be lower than it otherwise would have been. The effect of tendon slack on the resting twitch has been reported to be of minor influence on the relationship between the superimposed response and voluntary torque, at flexed knee angles (Behm et al. 2001; Bulow et al. 1993; Rutherford et al. 1986). However, tendon slack is greater at short compared to long muscle length (Muraoka et al. 2004). Therefore, particularly at short muscle lengths, scaling the superimposed torque response to the response obtained at rest may lead to a relatively lower calculated VA at short (extended knee angles) compared to long muscle lengths (flexed knee angles).

In subjects with a high ability for maximal VA, a neural drive may be reached that is usually unattainable for regular healthy subjects. Moreover, during voluntary fast isometric torque development, rectified surface EMG levels of the knee extensors may be substantially higher than at the torque plateau of an MVC (de Ruiter et al. 2004). These findings indicate a potential to increase neural drive at the torque plateau of a MVC (see also Herbert and Gandevia 1999). However, based on the shape of the stimulation frequency-force (de Ruiter et al. 1999; Deutekom et al. 2000) and pCa-force curve (Stephenson and Williams 1982) at high force levels large increases in neural drive and calcium are required for a comparatively small increase in force. As EMG is often measured to quantify the neural drive at different contraction intensities, this has important consequences for the EMG-torque relationship. EMG is generally normalized to the EMG obtained during MVC, a disproportionate increase in EMG at very high force levels will consequently lead to a more curvilinear EMG-torque relationship. However, this can only be demonstrated in subjects who are able to consistently reach high levels of neural drive. The shape of a subjects' EMG-torque relationship would therefore be determined by the subjects' ability for VA. This would explain why both linear (Woods and Bigland-Ritchie 1983) and non-linear (Alkner et al. 2000) EMG-torque relationships have been reported. Therefore, this study further aims to investigate the EMG-torque relationship of the knee extensors in subjects with high ability for VA.

\section{Methods}

\section{Subjects}

Seven healthy male subjects (23-32 year, $69-83 \mathrm{~kg}$, and $1.73-1.93 \mathrm{~m})$ volunteered to be subjects for this investigation. Before participation, each subject was thoroughly 
informed about the procedures and provided written informed consent prior to testing. Only subjects capable of $>95 \%$ VA across knee angles (see below) were included in the present study. The study was performed according to the Declaration of Helsinki and approved by the local ethics committee. Subjects did not perform any fatiguing exercise $48 \mathrm{~h}$ prior to measurements.

\section{Experimental procedures}

The subjects visited our laboratory on two occasions with at least 1 day in between. All subjects were familiar with the set-up, electrical stimulation, and isometric knee extension from previous studies (de Ruiter et al. 2004; Kooistra et al. 2005). During the first experimental day, several contractions with superimposed stimulation (see below) were performed at $30^{\circ}$ (extended) and 60 and $90^{\circ}$ (flexed) knee angles. At the same time, surface EMG of the rectus femoris (RF), vastus lateralis (VL), and vastus medialis (VM) was recorded. To gain information concerning test-retest variability, the experimental procedure performed on experimental day 1 was repeated during experimental day 2 .

\section{Torque measurements}

Isometric knee extension torque of the right leg was measured using a custom-built dynamometer (Kooistra et al. 2005). Subjects were seated with their hips at $70^{\circ}\left(0^{\circ}=\right.$ full extension). Shoulders, hips, and lower thigh were strapped to the dynamometer. By very tight strapping of the hips and lower thigh, a contribution of the hip extensors to knee extension was absolutely impossible. Moreover, during contractions, subjects had to lean forward about $10 \mathrm{~cm}$ with their shoulders (while grasping the strap around their shoulders) pushing their lower back into the back rest. This further guaranteed that no hip extension occurred. The distal part of the shank was strapped to a force transducer (KAP, E/200 Hz, Bienfait B.V. Haarlem, The Netherlands) that was attached to the lever arm of the dynamometer. A shin guard ensured subjects could exert maximal forces without discomfort at the shin. The compliance of the dynamometer at the position of the force transducer was $1.4 \times 10^{-4} \mathrm{deg} / \mathrm{Nm}$. The backrest, force transducer height, and its medio-lateral position were adjusted for precise alignment of the knee axis with the axis of rotation of the dynamometer arm. A crank enabled changing of the knee angle of the subject. Knee joint angles were determined with a handheld goniometer (model G300, Whitehall Manufacturing) using the greater trochanter and lateral epicondyle of the femur, and the lateral malleolus of the fibula as references. The dynamometer arm was positioned so that the indicated knee angles were angles in an active state, with subjects delivering $~ 50 \%$ of MVC. During pilot experiments using an electrogoniometer attached to the lateral side of the knee, the changes (active-passive) in knee angle were found to be $3-7^{\circ}$, independent of knee angle, with minimal $\left(<1^{\circ}\right)$ changes above $~ 50 \%$ of MVC. Real-time force applied to the force transducer was displayed on line on a computer monitor and digitally stored $(1 \mathrm{kHz})$ on computer disc. The force signals were automatically corrected for gravity at each angle: the average force applied by the weight of the limb to the transducer during the first $50 \mathrm{~ms}$ after the start of a recording, with the subject seated in a relaxed manner, was set to zero force by the computer program. Extension torque was calculated by multiplication of force with the individual lever arm and data will be presented as torque.

\section{Electrical stimulation}

A cathode (self-adhesive stimulation electrode, $5 \times 5 \mathrm{~cm}$, Schwa-Medico, The Netherlands) was placed over the femoral nerve. The anode $(13 \times 8 \mathrm{~cm})$ was placed over the gluteal fold. The quadriceps femoris muscle was stimulated transcutaneously with rectangular pulses of $100 \mu$ s using a computer-controlled constant current stimulator (Digitimer DS7H, Digitimer Ltd., Welwyn Garden City, UK). Stimulation current was increased until torque measured in response to a triplet (three $100 \mu$ s pulses applied at $300 \mathrm{~Hz}$ ) levelled off at each knee angle tested. The current (in $\mathrm{mA}$ ) was then increased by a further $50 \mathrm{~mA}$ to ensure supramaximal stimulation (range: $250-600 \mathrm{~mA}$ ). At this point, we considered all muscle fibres of the knee extensors to be activated. Pilot studies had shown that triplet torque did not increase after an MVC at any knee angle, indicating that any potential potential post-activation potentiation did not result in a triplet torque increase (see also Discussion). Usually, torque generated by the triplet is about $\sim 35 \%$ of the MTC (Kooistra et al. 2005). Furthermore, in pilot studies, the stimulation current was increased far beyond supramaximal levels (up to $200 \%$ ) without a subsequent decrease in triplet torque. Hence, the hamstring muscles were assumed not to be activated during supramaximal stimulation of the knee extensors (see also Discussion).

\section{Voluntary activation}

During experimental day 1 , the level of maximal VA was determined in random order at $30^{\circ}, 60^{\circ}$, and $90^{\circ}$ knee angles. At each knee angle, subjects were asked to maximally generate isometric torques for about $3-4 \mathrm{~s}$ to determine MVC extension torque. Real-time torque was visible on a computer monitor and subjects were vigorously encouraged to exceed their maximal value, which was also displayed. MVC torque was defined as highest torque within a 
stable $1,000 \mathrm{~ms}$ plateau of the torque signal. MVC torque was taken as the highest value, which did not exceed preceding attempts by $>5 \%$, allowing a maximum of four attempts to prevent fatigue. In the incidental case a trial had no stable plateau, the trial was repeated. Subjects then performed a series of superimposed measurements consisting of a triplet applied to a fully relaxed muscle and another triplet superimposed on the stable part of the torque signal (Fig. 1). If the torque level was unstable ( $>1 \%$ torque fluctuation) prior $(100 \mathrm{~ms})$ to stimulation, the contraction was repeated. As all our subjects were very experienced at maintaining stable torque levels at all contraction intensities, it was rarely necessary to repeat a measurement. As an indication, for all subjects, less than 1 in 10 contractions (across all knee angles and intensities) were repeated. Several different contraction intensities with an emphasis toward higher intensities were chosen $(25,50$, $70,80,90$, and 100\%MVC). At each knee angle, the order of the contraction intensities with superimposed triplet was randomized, and the order of the knee angles was randomized per subject. A 3-min rest period separated contractions. For each superimposed contraction, the accepted formula for the interpolated twitch torque technique (Allen et al. 1995; Babault et al. 2001, 2006; Becker and Awiszus 2001; de Ruiter et al. 2005; Kooistra et al. 2005; Maffiuletti and Lepers 2003; McKenzie et al. 1992; Newman et al. 2003; Oskouei et al. 2003) was used to determine the level of voluntary activation (VA):

$\mathrm{VA}(\%)=100$

$-[$ (triplet torque increment $/$ resting triplet $) \times 100]$

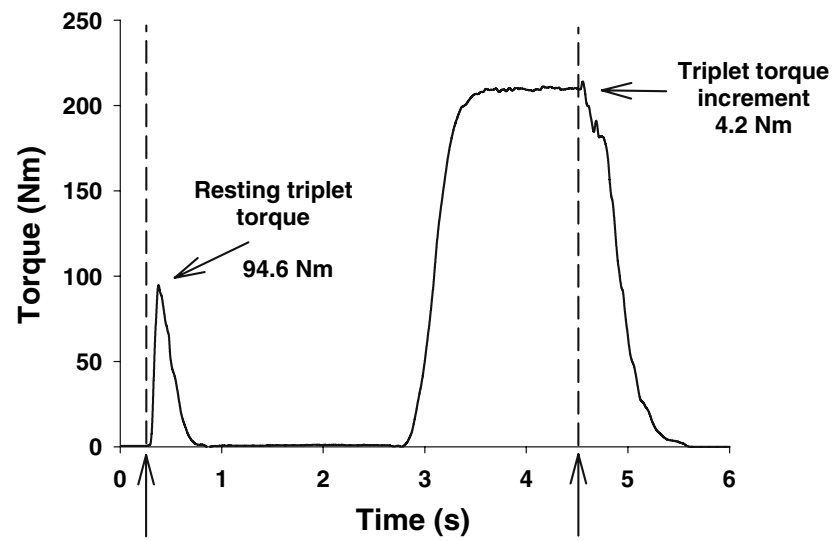

Fig. 1 Typical example of a superimposed contraction. The resting triplet torque as a result of the electrical stimulation as well as the triplet torque increment on a $100 \% \mathrm{MVC}$ contraction at the $90^{\circ}$ knee angle are shown. The timing of the triplet stimulation is shown by the vertical arrows and vertical dashed lines. Voluntary activation (VA) is generally calculated using the following equation: VA $(\%)=100$ [(triplet torque increment/resting triplet torque $) \times 100$ ]. In this particular example, voluntary activation was calculated to be: 100 $[(4.2 / 94.6) \times 100]=95.6 \%$
With this method, the triplet torque increment is expressed as a percentage of the resting triplet torque, and subtraction from $100 \%$ results in a value for VA (Fig. 1). Maximal VA was obtained from the $100 \%$ MVC superimposed contraction. To illustrate the relationship between superimposed and voluntary torque from 90 to $100 \% \mathrm{VA}$, only subjects capable of an average maximal VA of $>95 \%$ across knee angles were included in the present study. MVC torques as close to the muscles' MTC as possible were thus obtained. Due to our strict inclusion criteria (average VA of $>95 \%$ ) at each knee angle, we were able to select a contraction at a lower than maximal torque level where the superimposed triplet was $\sim 10 \%$ of resting triplet. At this torque level, VA is conventionally calculated to be $\sim 90 \%$, and we expressed this torque level as a percentage of MVC. We could thus compare the theoretical $10 \%$ torque increase implied by a conventionally calculated VA level of $90 \%$ with the torque increase actually achieved by our subjects during MVC. This provides us with a good indication of the potential torque increase that may be possible in regular healthy subjects with a maximal VA level of $\sim 90 \%$. The $\sim 90 \%$ is a VA level that is frequently reported in the literature for regular healthy subjects (Babault et al. 2001, 2003; Bulow et al. 1993; de Ruiter et al. 2004; Newman et al. 2003; Oskouei et al. 2003; Suter and Herzog 1997).

Furthermore, for each subject and for each superimposed contraction, the increment in torque as a result of the superimposed triplet was plotted as a function of the torque reached just prior to the superimposed stimulation (e.g. Figs. 2, 3a). Curvilinear relationships between voluntary and superimposed torque are often reported in the literature for group data (e.g. Behm et al. 2001; Dowling et al. 1994; Oskouei et al. 2003; Suter and Herzog 1997). In the present study, however, neither a linear nor a curvilinear fit closely followed the data points for any of the individual subjects at any knee angle. However, linear regression was performed on the highest four contraction intensities (70, 80, 90 , and $100 \%$ ), and $R^{2}$ values were calculated at each knee angle for each subject. This was done to show that although superimposed torque increments were small at high contraction intensities, the torque increment continued to decrease with contraction intensity (e.g. Fig. 3a, b).

Surface electromyography

Electromyographic activity of the RF, VL, and VM muscles was recorded using surface EMG electrodes (Blue Sensor, Ambu, Ølstykke, Denmark, lead-off area: $1.0 \mathrm{~cm}^{2}$ ). After shaving, roughening, and cleansing the skin with $70 \%$ ethanol, electrodes were placed on the muscle belly in a bi-polar configuration in line with the muscle fibre direction with a centre to centre inter-electrode 


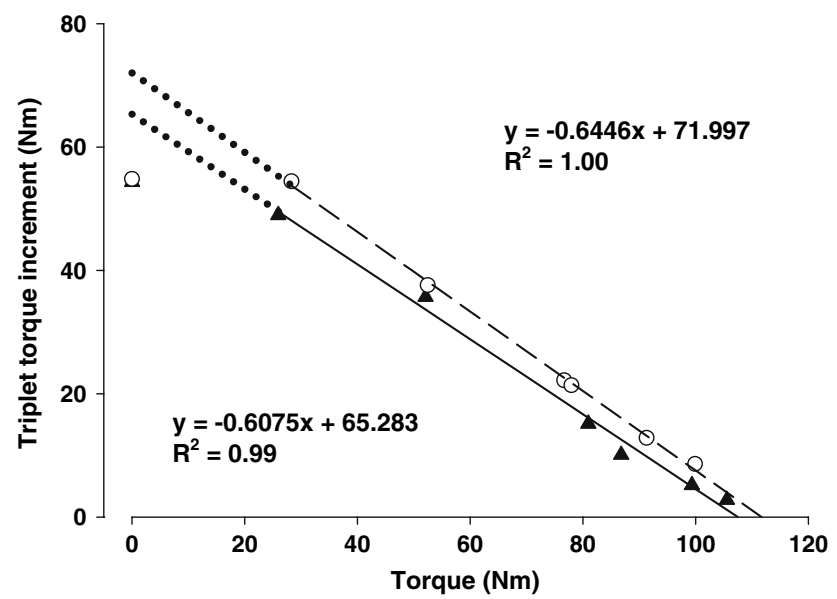

Fig. 2 Torque increment as result of the triplet versus voluntary torque delivered at the $30^{\circ}$ knee angle by a normal healthy subject in a pilot study. The empty circles denote the first day of testing, and the black triangles the second day. The dashed and solid lines represent linear regression fits for the data points on experimental days 1 and 2, respectively, excluding the resting triplet torque at $0 \mathrm{Nm}$. The resting triplet torque is clearly underestimated as it is very similar to the triplet torque increment at $\sim 30 \mathrm{Nm}$. The dotted lines represent backward extrapolation of the regression fits for both experimental days and illustrate the underestimation of the resting triplet torque as a result of tendon slack. For each experimental day, the respective equations and $R^{2}$ values are shown. Using the interpolated twitch torque technique, voluntary activation (VA) is calculated to be quite high for experimental days $1(84.4 \%)$ and $2(94.7 \%)$

distance of $25 \mathrm{~mm}$. Reference electrodes were placed on bone structures, on each patella, and on the lateral epicondyle of the femur of the right leg. Surface EMG signals were amplified $(\times 100)$, digitized $(1 \mathrm{kHz})$, and stored with the force signal on computer disc. All EMG signals were band-pass filtered $(10-400 \mathrm{~Hz})$. Rectified surface EMG amplitude (rsEMG) was calculated for the RF, VL, and VM for 1,000 ms segments just before the superimposed stimulation. RsEMG values obtained during the highest torque level on each experimental day were set to $100 \%$.

\section{Statistics}

All results are presented as mean (SD. Knee angle effects were tested for significance with repeated measures ANOVA. If significant main effects were observed, Bonferroni tests were performed for post hoc analysis. Test-retest reliability was tested for using the intraclass correlation coefficient. The level of significance of all statistical analyses was set at $P<0.05$.

\section{Results}

Torque levels during the superimposed contractions at the $25,50,70,80,90$, and $100 \%$ MVC levels were not different
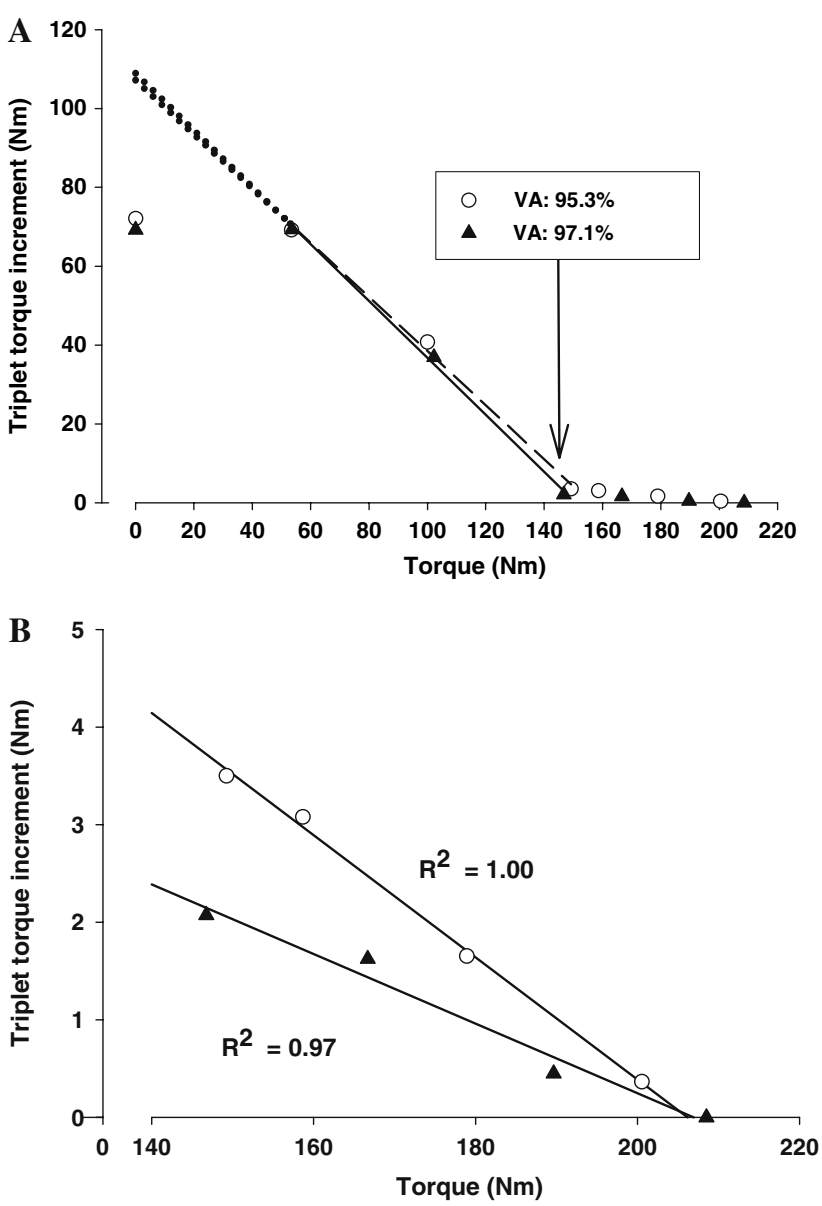

Fig. 3 a The torque increment as a result of the triplet on a voluntary contraction ( $y$-axis) is shown as a function of the voluntarily delivered torque just before the triplet ( $x$-axis). The data points shown are those of subject no. 1, a subject with high ability for maximal voluntary activation, at the $30^{\circ}$ knee angle. The open circles denote results from experimental day 1 and the filled triangles from experimental day 2 . It is immediately apparent that using a linear or a curvilinear fit over all data points is incorrect for this subject. The dashed and solid lines illustrate linear regression fits that have been performed for a voluntary torque level of $\sim 50-150 \mathrm{Nm}$ for experimental days 1 and 2 , respectively. The dotted lines represent backward extrapolation of the regression fits for both experimental days and illustrate the potential effect of tendon slack on the resting triplet torque, especially when compared to the triplet torque increment at $\sim 60 \mathrm{Nm}$. For clarity, and to illustrate the continued decrease of the triplet torque increment with increasing voluntary torque, the data points of torque levels above $140 \mathrm{Nm}$ have been replotted in Fig. 3b. b Data points from Fig. 3A have been replotted for torque levels above $140 \mathrm{Nm}$. The torque increment as a result of the triplet is shown as a function of the voluntarily delivered torque just before the triplet ( $x$-axis) for four high intensity contractions (70, 80, 90, and 100\%MVC). The open circles represent data points obtained on experimental day 1 and the filled triangles experimental day 2. A linear regression line shows the continued decrease in triplet torque increment with increase in contraction intensity for each set of four data points with corresponding $R^{2}$ values

from the intended values or different between experimental days. Linear extrapolation of the torque increment as a result of the triplet on the high intensity contractions (70, 
80,90 , and $100 \% \mathrm{MVC}$ ) provided high $R^{2}$ values at each knee angle $\left(30^{\circ}: 0.89 \pm 0.09 ; 60^{\circ}: 0.90 \pm 0.10\right.$; and $90^{\circ}$ : $0.87 \pm 0.10$ averaged over days), which were not different between knee angles or the two experimental days (e.g. Fig. 3b). The continuing consistent decrement of the triplet torque increment with increase in contraction intensity was denoted by the overall negative slope $(-0.13 \pm 0.05$ across days and angles), which was not different between days and angles. The relationship between the triplet torque increment and voluntary torque is shown in Fig. 4 for the 30,60 , and $90^{\circ}$ knee angles.

There was no difference in resting triplet torque or MVC between experimental days and average values across days are presented. At the $60^{\circ}$ knee angle, MVC was significantly $(P<0.05)$ greater compared to both 30 and $90^{\circ}$ knee angles (Table 1). Resting triplet torque, however, was significantly $(P<0.05)$ lower at $30^{\circ}$ compared to 60 and

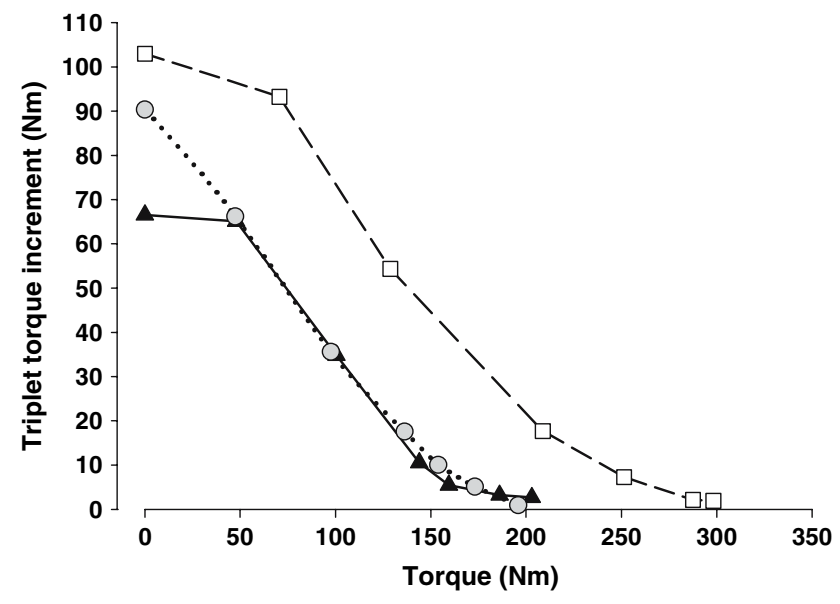

Fig. 4 The torque increment as result of the triplet is shown as a function of voluntary torque for subject no. 5 on experimental day 1 . Note that at the higher contraction intensities $(>70 \%)$, the shape of the curve is similar at the $30^{\circ}$ (black triangles, solid line), $60^{\circ}$ (white squares, dashed line), and $90^{\circ}$ (grey circles, dotted line) knee angle at the higher $(>70 \% \mathrm{MVC})$ contraction intensities $90^{\circ}$ (Table 1). Reproducibility for both MVC and resting triplet torque was very high, which was illustrated by high ICC values (Table 1).

\section{Voluntary activation}

Overall, there was no difference in maximal VA, determined by applying the ITT to the highest MVC, between experimental days and average values across days are presented. The significantly lower triplet torque at $30^{\circ}$ compared to the $90^{\circ}$ knee angle did not result in a lower maximal VA at $30^{\circ}$ compared to $90^{\circ}$ At each knee angle, maximal VA was very high and similar between knee angles (Fig. 5). Furthermore, at each knee angle, at torques where the triplet increment was $\sim 10 \%(8.5 \pm 2.6 \%)$, VA was calculated to be $\sim 90 \%(91.5 \pm 2.6 \%$, across angles $)$. This VA level turned out to be significantly higher compared with the relative voluntary torque level at which the triplet was superimposed. The torques at which VA was calculated to be $91.5 \pm 2.6 \%$ represented only 79.6 ( $6.1 \%$ of MVC (across knee angles), a finding that was similar at each knee angle $(P>0.05)$. Note that at this torque level, the triplet torque increments were already small $(3.2 \pm 1.1 \% \mathrm{MVC})$ compared to the torque generated when the triplet was applied on the resting muscle ( $\sim 37 \% \mathrm{MVC})$. The key point we want to address with our study is illustrated in Fig. 6. For both subject nos. 1 and 7, a VA level of $\sim 90 \%$ was calculated at the $60^{\circ}$ knee angle at a torque level of $\sim 250 \mathrm{Nm}$. This suggests a potential further increase of maximal torque by $10 \%$. However, subject no. 1 was able to significantly increase his torque production by more than $30 \%$.

\section{EMG}

The rsEMG values obtained during MVC were similar between days at each knee angle $(P=0.96)$. For all submaximal contraction intensities, normalized rsEMG values for each muscle (RF, VL, and VM) at each contraction

Table 1 Torque

\begin{tabular}{lllll}
\hline & \multicolumn{2}{l}{ Knee angle } & & \\
\cline { 2 - 5 } & $30^{\circ}$ & $60^{\circ}$ & $90^{\circ}$ & $189.6 \pm 19.7$ \\
\hline MVC $(\mathrm{Nm})$ & $186.1 \pm 36.6$ & $278.4 \pm 44.5^{*}$ & $80.1 \pm 10.8$ & $0.99 * * *$ \\
Triplet torque $(\mathrm{Nm})$ & $65.9 \pm 10.0^{* *}$ & $91.6 \pm 15.8$ & $0.96^{* * *}$ \\
\hline
\end{tabular}

All values are means $\pm \mathrm{SD}$, averages across days shown, $n=7$. Maximal voluntary isometric knee extension (MVC) and resting triplet torque for the $30^{\circ}, 60^{\circ}$, and $90^{\circ}$ knee angle

* Significantly different from $30^{\circ}$ to $90^{\circ}$ knee angle $(P<0.05)$

** Significantly different from $60^{\circ}$ to $90^{\circ}$ knee angle $(P<0.05)$

*** Significant ICC, the intraclass correlation coefficient (ICC) was calculated for the measurements made on experimental days 1 and 2 and is shown in the last column 


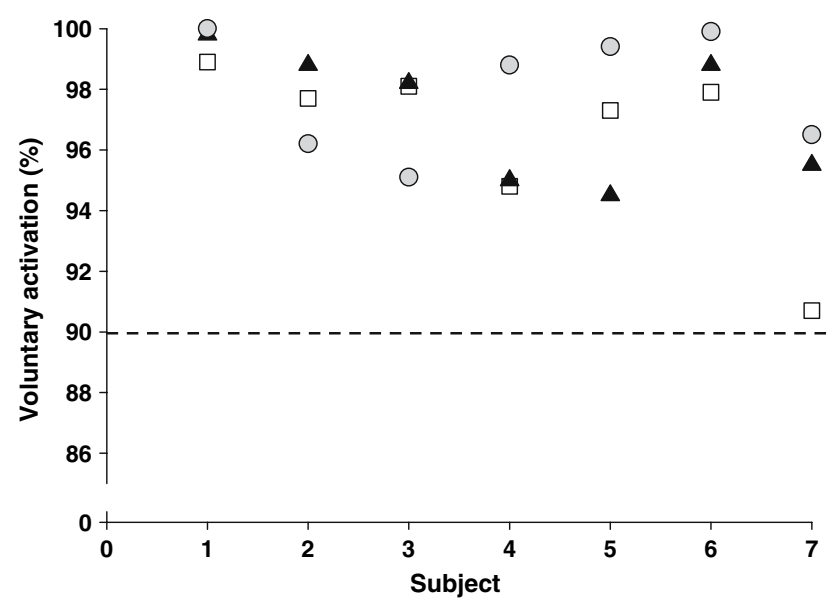

Fig. 5 Average (2 days) maximal voluntary activation (VA) per subject for the $30^{\circ}$ (black triangles), $60^{\circ}$ (white squares), and $90^{\circ}$ (grey circles) knee angles. Note that only 2 out of a total of 21 data points are below 95\%VA. The horizontal dotted line indicates the $90 \%$ VA level that is generally obtained for the knee extensors by regular healthy subjects

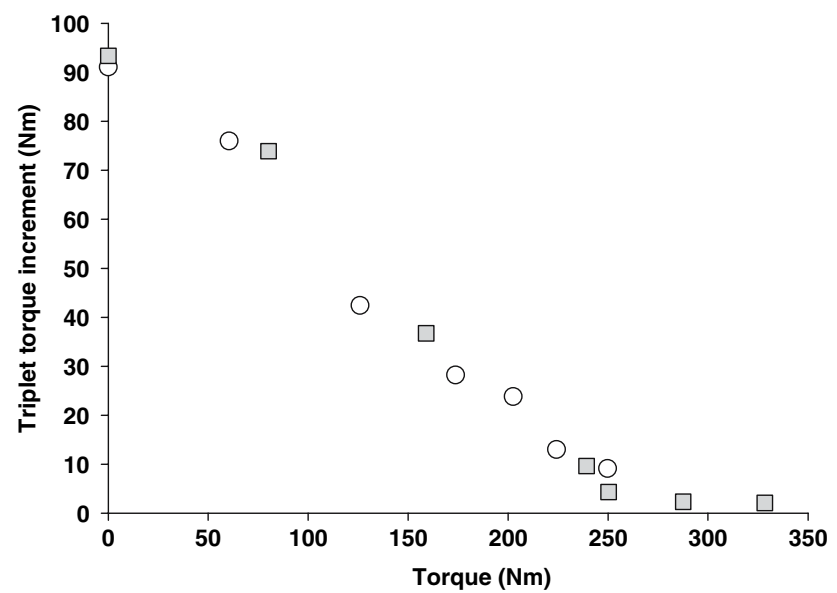

Fig. 6 The torque increment as result of the triplet is shown as a function of voluntary torque for subject no. 1 (grey squares) and 7 (open circles) at the $60^{\circ}$ knee angle on day 1 . At the $60^{\circ}$ knee angle, the maximal VA level of subject no. 7 calculated at $250 \mathrm{Nm}$ was $90.7 \%$, which resembled that of a regular subject, and is exceptionally low in our study (see lowest point in Fig. 5). A VA of $90.0 \%$ is also calculated at $240 \mathrm{Nm}$ for subject no. 1 . In both subjects, this implies a further potential increase in torque of $10 \%$, yet a $>30 \%$ torque increase (to $330 \mathrm{Nm}$ ) could be demonstrated in subject 1 . This is most likely due to the exceptional neural drive of subject 1 ( $98 \% \mathrm{VA})$ during his best attempts at this knee angle

intensity was similar between days; hence, averaged values across days are presented. Furthermore, no difference between knee angles or muscles was found, and with each increase in contraction intensity, there was a significant increase in normalized rsEMG (Fig. 7a, VL shown). An increase of normalized rsEMG of $\sim 21 \%$ (across angles and muscles) was seen for a $25 \%$ increase in torque from
25 to $50 \%$ MVC (Fig. 7a). When contraction intensities approached MVC, there was a much larger increase in normalized rsEMG $(\sim 34 \%)$ relative to the torque increase of only $\sim 18 \%$ at all knee angles (Fig. 7a). In regular healthy subjects, the highest MVC would have occurred at torque levels where VA would have been calculated to be $\sim 90 \%$, and consequently the EMG-torque relationship would most likely have been considerably less curvilinear. This is illustrated by the two EMG-torque relationships shown in Fig. $7 \mathrm{~b}$ for the VL at the $90^{\circ}$ knee angle. The first EMG-torque relationship includes all contraction intensities measured, whereas for the second EMG-torque relationship, the 90 and $100 \% \mathrm{MVC}$ contraction intensities have been removed. In the latter case, all rsEMG values are normalized to a torque level that corresponds to the maximal VA level of $~ 90 \%$ (generally obtained by regular healthy subjects), the result being a near linear EMG-torque relationship (Fig. 7b).

As a consequence of the disproportionate increase in EMG as MVC is approached, normalized rsEMG values obtained at intensities below MVC are normalized to a relatively large value. Hence, these normalized rsEMG values are relatively smaller compared to the relative torques at which they have been obtained. For example, at 25 and $50 \% \mathrm{MVC}$, normalized rsEMG for the RF, VL, and VM muscle was significantly less than would be expected based on the \%MVC at which they were obtained at all knee angles (Fig. 7a, VL shown). By normalizing the rsEMG levels to the rsEMG values obtained at a torque level that corresponds to the VA level of $90 \%$ as found in regular healthy subjects, normalized rsEMG values are closer to the line of identity, and a more linear EMGtorque relationship is seen (Fig. 7b).

\section{Discussion}

In the present study, the relationship between calculated VA and voluntary torque was assessed in subjects with high ability for maximal VA at different knee angles. At the same time, the EMG-torque relationship in these selected subjects was examined. The first and main finding of the present study was that when VA is calculated to be $\sim 90 \%$ (as in regular healthy subjects), this probably represents a considerable overestimation of the subjects' ability to maximally drive their quadriceps muscles. An additional finding was that, although resting triplet torque was lower at $30^{\circ}$ versus $90^{\circ}$, this was of minor influence on the calculated maximal VA in our subjects. Furthermore, a relatively large increase in normalized rsEMG was observed as MVC was approached, making the shape of the normalized EMG-torque relationship of the knee extensors curvilinear. 

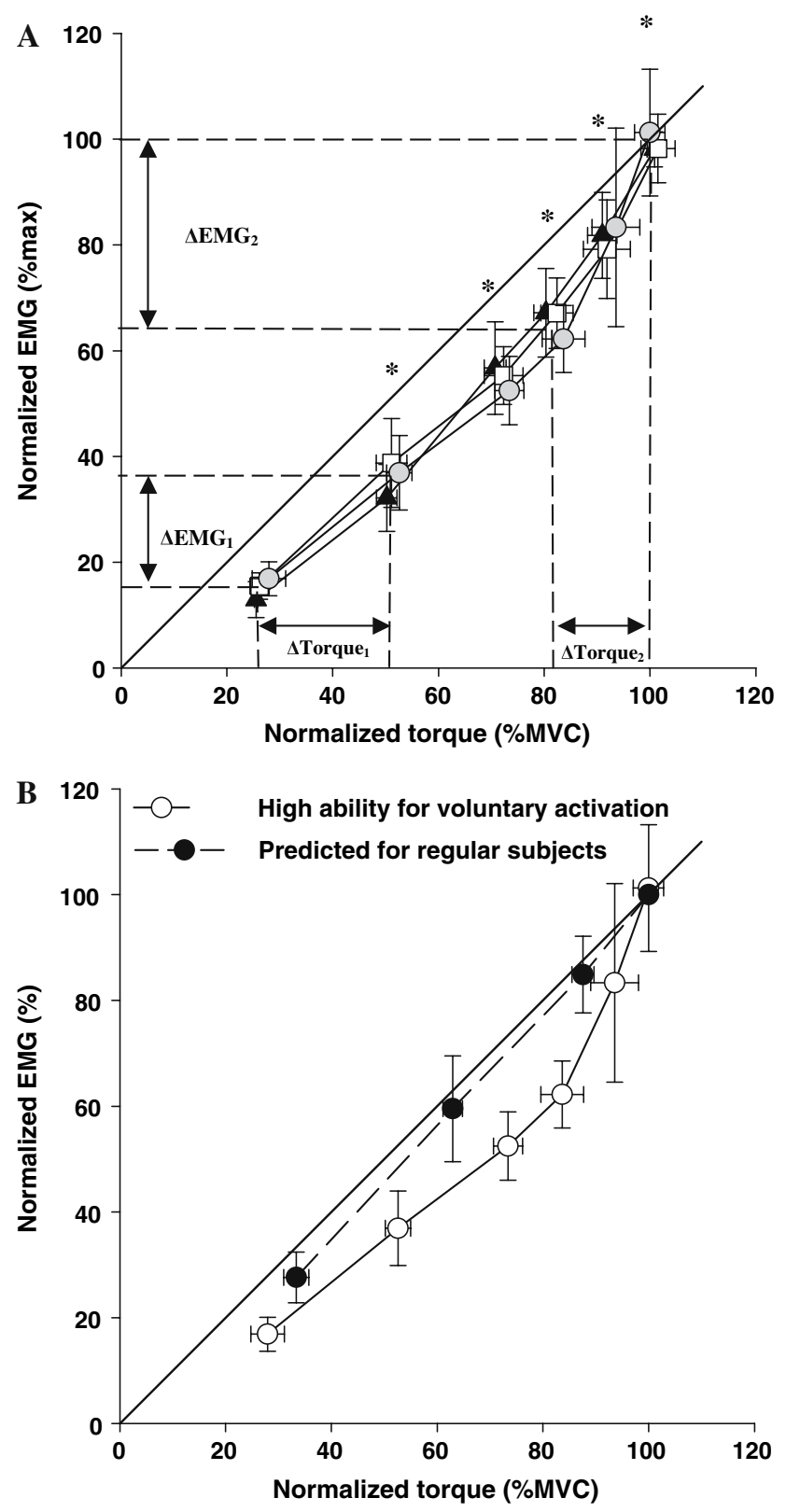

Maximal voluntary activation and knee angle

Subjects in the present study exhibited a consistent high level of maximal VA of the knee extensors (Fig. 5), which is higher than the maximal VA levels of $90 \%$ that are generally reported for the knee extensors in regular healthy subjects when applying the ITT (Babault et al. 2001, 2003; Bulow et al. 1993; de Ruiter et al. 2004; Newman et al. 2003; Oskouei et al. 2003; Suter and Herzog 1997). Although full activation of the knee extensors has been reported in the past (Bigland-Ritchie et al. 1986; Rutherford et al. 1986), in those studies a single superimposed twitch was used. Due to the declining signal-to-noise ratio with increase in contrac-
Fig. 7 a Normalized rsEMG levels averaged over the two experimental days versus normalized torque for the vastus lateralis (VL) muscle at $30^{\circ}$ (black triangles), $60^{\circ}$ (white squares), and $90^{\circ}$ (grey circles $)$ knee angles. *Significantly different $(P<0.05)$ from preceding intensity level. At the lower contraction intensities, $\triangle$ EMG1 denotes the increase in EMG (21\%, across muscles) that is accompanied by a comparatively larger increase torque $(\sim 25 \%)$, which is denoted by $\Delta$ Torque $_{1}$. Conversely, $\triangle$ EMG2 denotes the much larger increase in EMG $(\sim 34 \%$, across muscles) that is accompanied by a comparatively smaller increase in normalized torque $\left(\sim 18 \%\right.$, denoted by $\Delta$ Torque $\left._{2}\right)$ as the contraction intensity approaches MVC. Note that on average for $\Delta$ Torque $_{2}$, calculated voluntary activation (VA) increased from $91.5 \pm 2.6 \%$ to $97.2 \pm 2.3 \%$. Thus, a $34 \%$ increase in normalized rsEMG was accompanied by an $18 \%$ increase in torque, for which only a $\sim 5.7 \%$ increase in VA (denoted by $\triangle \mathrm{VA}$ ) was calculated. As a consequence of the relatively large increase in EMG as MVC is approached, the rsEMG of contraction intensities below MVC are normalized to a relatively large value and are located well beneath the line of identity. b Normalized rsEMG levels averaged over days versus normalized torque for the vastus lateralis (VL) muscle at the $90^{\circ}$ knee angle. The white circles represent rsEMG values that have been normalized to the MVC of subjects with a very high ability for voluntary activation (VA). With the black circles, the EMG-torque relationship for regular healthy subjects has been predicted. The black circles denote rsEMG values that have been renormalized to the rsEMG value reached at $90 \%$ VA. This is similar to the usual maximal VA in regular individuals. Note that the EMG-torque relationship predicted for regular healthy subjects is linear and closer to the line of identity compared to subjects with very high ability for voluntary activation

tion intensity, the detection of a single superimposed twitch is difficult, and $100 \%$ VA may mistakenly have been assumed (Dowling et al. 1994). To improve the signal-to-noise ratio, multiple stimuli have been suggested (Gandevia and McKenzie 1988; Suter and Herzog 2001). In the present study, triplet stimulation provided considerable resting triplet torque levels at each knee angle (Table 1), which ensured a good signalto-noise ratio. Furthermore, pilot studies had shown no increase in triplet torque before and after an MVC at any knee angle. By using supramaximal and high frequency $(300 \mathrm{~Hz})$ triplet stimulation, we avoided the potential influence of post-activation potentiation on our data.

A limitation of the ITT may be the scaling of the twitch increment to the resting twitch. When muscle is stimulated by a twitch at rest, all tendon slack has to be taken up; conversely, for a twitch superimposed on an ongoing isometric contraction, no further slack has to be taken up (Suter and Herzog 1997). Since calculated VA is determined by the scaling of the triplet torque increment to the resting triplet, calculated VA will be reduced by the influence of tendon slack. As the effect of tendon slack is greater at short versus long muscle length (Muraoka et al. 2004), VA will be influenced to a greater degree at short versus long muscle length. Besides tendon slack, length-dependent $\mathrm{Ca}^{2+}$ sensitivity may also influence the size of the resting triplet by 
shifting the resting triplet torque-length relationship to shorter muscle length compared to the MVC torquelength relationship. The length-dependent effect of tendon slack and $\mathrm{Ca}^{2+}$ sensitivity were reduced by the use of a triplet (Kawakami et al. 2001), but not abolished as triplet torque was significantly smaller at the $30^{\circ}$ versus $90^{\circ}$ knee angle (Table 1), whereas MVC levels were similar. This implies that despite using triplet stimulation, compared to the $\mathrm{MVC}$, resting triplet torque remains relatively lower at the $30^{\circ}$ versus $90^{\circ}$ knee angle. The latter is supported by Figs. 2 and 3A, where the triplet response is similar at 0 and $25 \% \mathrm{MVC}$ at the $30^{\circ}$ knee angle. It is evident that calculated VA will be influenced by tendon slack, as the resting triplet is influenced by tendon slack, especially at short muscle length, whereas the triplet torque increment is not (Suter and Herzog 1997).

Despite a lower resting triplet torque at the $30^{\circ}$ compared to the $90^{\circ}$ knee angle, maximal VA was not different between the $30^{\circ}$ and $90^{\circ}$ knee angle in our subjects. From the literature, it is unclear whether maximal VA is knee angle-dependent. Kubo et al. (2004) report a lower level of maximal VA at extended compared to flexed knee angles, whereas Suter and Herzog (1997) find maximal VA to be highest at the most extended knee angle $\left(15^{\circ}\right)$. Newman et al. (2003) and Babault et al. (2003), however, find no effect of knee angle on maximal VA, which is in line with our current findings. Note that due to our selection criteria (average maximal VA of $95 \%$, and at least $90 \%$ at each knee angle), maximal VA was very high $(\sim 97 \%)$ and close to $100 \%$. Potential knee angle-dependent differences in maximal VA could consequently only occur within a very small range. Furthermore, the small size of the superimposed triplet torque makes the calculation of maximal VA insensitive to large differences in the size of the resting triplet torque. By hypothetically increasing the resting triplet torque at $30^{\circ}$ by $20 \%$, making it similar to the $90^{\circ} \mathrm{knee}$ angle, an increase in maximal VA of less than $0.5 \%$ is calculated at the $30^{\circ}$ knee angle. In subject populations possessing lower maximal VA levels (e.g. patient groups), a relatively greater increment will be obtained on an MVC. This greater increment is more susceptible to the muscle-length dependent influence of, for example, tendon slack on the size of the resting triplet. The influence of tendon slack would have been greater still had a twitch instead of a triplet been used (Kawakami et al. 2001), leading to an even greater difference in resting twitch size at $30^{\circ}$ versus $90^{\circ}$. Combined with the use of a resting twitch, therefore, a muscle length-dependent effect of tendon slack is expected to significantly influence the calculation of VA in this subject population.
Calculated voluntary activation in relation to relative voluntary torque

For the most accurate assessment of maximal VA, Behm et al. (1996) recommended using the MVC with a superimposed electrical stimulus. As mentioned previously, in regular healthy subjects a maximal VA level of $\sim 90 \%$ is often reported for the knee extensors using this method (Babault et al. 2001, 2003; Bulow et al. 1993; de Ruiter et al. 2004; Newman et al. 2003; Oskouei et al. 2003; Suter and Herzog 1997) implying that the MTC has almost been reached. In the present study, with our selected subject group, we were able to demonstrate that $90 \% \mathrm{VA}$ is already calculated at torque levels that represent only $\sim 79 \%$ of MVC (across knee angles). In some subjects, this phenomenon is very pronounced. For the subject shown in Fig. 3a and b, already at a torque of $\sim 148 \mathrm{Nm}$, VA using the ITT was calculated to be 95.3 and $97.1 \%$ for experimental day 1 and 2, respectively. These are very high VA levels that have been calculated at $148 \mathrm{Nm}$, especially when compared with the MVC of $>200 \mathrm{Nm}$ that was obtained on both experimental days. Note that the higher part of the curve (in this example over $140 \mathrm{Nm}$ ), as shown in Fig. 3 b can usually not be obtained in regular healthy subjects. In our experience, we can only demonstrate the existence of this part of the curve in subjects with a very high ability for maximal VA ( $>99 \%$ in this extreme example, subject no. 1 in Fig. 5). This finding strongly suggests that the $90 \% \mathrm{VA}$ reported for regular healthy subjects is a large underestimation of the MTC of the muscle. A far greater torque increase therefore seems possible than the $10 \%$ implied by a calculated VA of $90 \%$ (Fig. 6). The main new finding, therefore, is that the present study for the first time quantifies the difference between calculated VA and relative voluntary torque.

Experimental and methodological factors

When relating the triplet torque increment to the triplet torque obtained at rest, it is important to maintain supramaximal stimulation during both conditions. In a previous study examining superimposed stimulation of the knee extensors (Behm et al. 1996), the authors reported significantly lower M-wave amplitudes during a superimposed stimulation on an MVC compared to during rest. They presumed that the contraction of the knee extensors resulted in a displacement of the stimulating electrode from its optimal position over the femoral nerve. In pilot studies of the present investigation, M-wave amplitude at rest and superimposed on an MVC was similar. Moreover, a substantial $(50 \%)$ increase in stimulation current did not lead to an increase in the size of the triplet increment on high intensity contractions (70 and $80 \% \mathrm{MVC}$ ). This strongly 
suggests that even during high intensity contractions, there was supramaximal stimulation of the femoral nerve during superimposed triplet stimulation. By using multiple stimuli during superimposed stimulation, spinal reflexes may have more time to diminish the superimposed response (Herbert and Gandevia 1999; Herbert et al. 1997). Modeling suggests that the use of twin stimuli at $100 \mathrm{~Hz}$ has a minimal effect on the estimates of VA (Herbert and Gandevia 1999). By applying a stimulation frequency of $300 \mathrm{~Hz}$ in the present study, triplet stimulation occurred within $10 \mathrm{~ms}$, which is even less when compared to the use of twin stimuli at $100 \mathrm{~Hz}$. The influence of spinal reflexes on the superimposed stimulation is therefore considered negligible. Furthermore, although the current passes through the sciatic nerve that innervates the hamstring muscles, its stimulation was regarded negligible. This is illustrated by the very small EMG recording of the m. biceps femoris compared to the VL muscle during stimulation of the knee extensors [Fig. 8, data from a previous study of ours (Kooistra et al. 2005)]. We accordingly assumed a marginal activation, if at all, of the hamstring muscles during superimposed stimulation and therefore no influence on our calculation of VA. In a previous study by our group (de Ruiter et al. 2004), we measured the contribution of coactivation during isometric knee extensions during brief MVCs and found it to be very small (5-10\%). As this finding is in accordance with previous findings (Newman et al. 2003; Psek and Cafarelli 1993), we did not measure coactivation in the present study and considered it minimal.

\section{The EMG-torque relationship}

As expected, when MVC was approached we observed a relatively large increase in normalized rsEMG (Fig. 7a). This large increase makes the shape of the EMG-torque relationship of the knee extensors curvilinear. This is in contrast to the findings of Woods and Bigland-Ritchie (Woods and Bigland-Ritchie 1983) who report a linear EMG-torque relationship, yet in accordance with Alkner et al. (2000) and Pincivero and Coelho (2000) who show a non-linear relationship for the knee extensors.

Based on a modelling study of motor-unit pools, Fuglevand et al. (1993) indicate that the difference between muscles that exhibit the linear as opposed to the nonlinear form of the EMG-torque relationship may be related to differences in firing rate behaviour rather than due to differences in recruitment organization. It is reasonable to expect that only subjects with a high ability for maximal VA (high neural drive) will achieve very high motor unit firing rates. For this large increase in firing rate, however, little gain in torque may be predicted (Herbert and Gandevia 1999). The large increase in excitation, as represented by the disproportionate increase in normalized

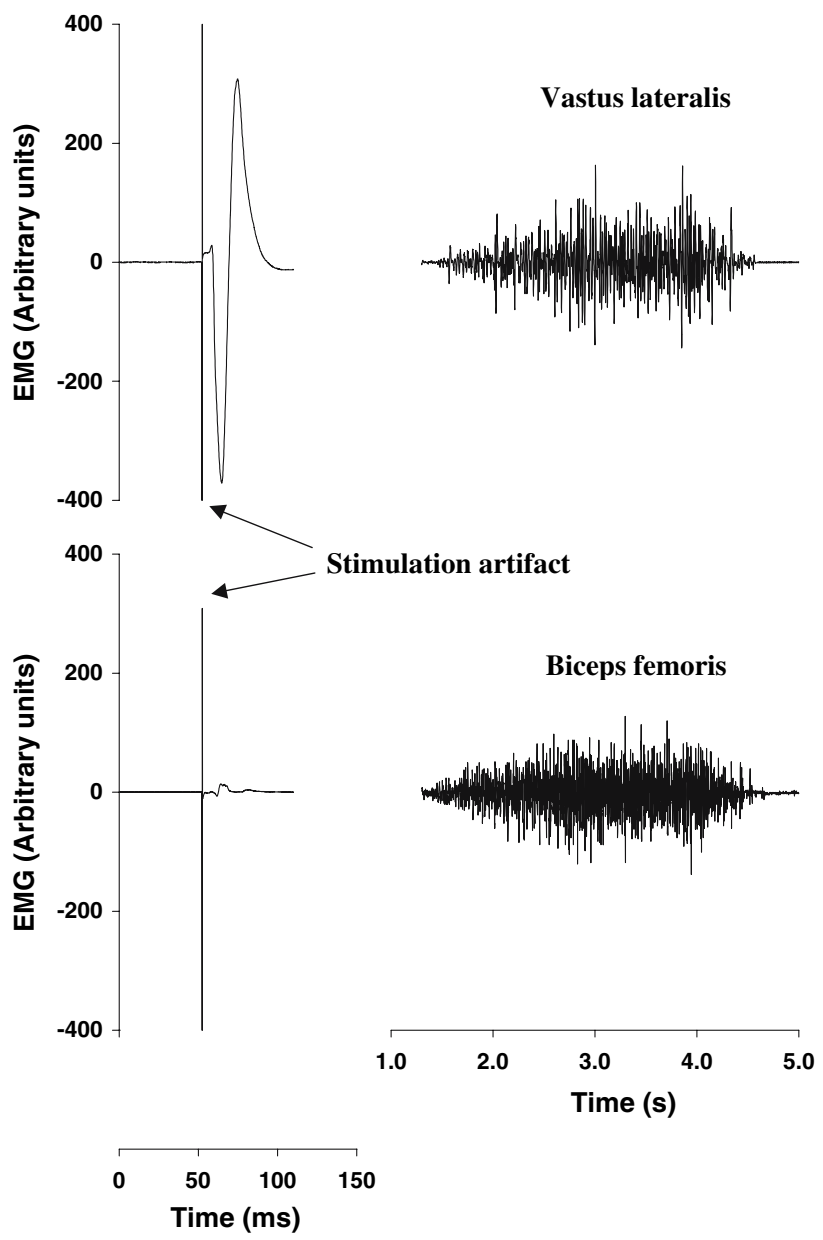

Fig. 8 Representative EMG recordings are shown for the vastus lateralis (VL, upper panel) and biceps femoris (BF, lower panel) muscle. On the left, the M-wave for the VL as a result of supramaximal twitch stimulation applied to the n. femoralis. The simultaneous EMG recording for the BF muscle is shown in the lower left panel. On the right, the EMG recording during maximal voluntary extension (top, right) and flexion (lower, right) torque is shown to illustrate that the lower $\mathrm{M}$-wave of the $\mathrm{BF}$ is not due a lower sensitivity of the $\mathrm{BF}$ recordings compared to the $\mathrm{VL}$ recordings

rsEMG when MVC was approached, confirms the model study by Herbert and Gandevia (1999). However, in contrast to their model where essentially $100 \% \mathrm{MVC}$ was achieved at $60 \%$ of maximal voluntary excitation, in the present study, we experimentally determined that the disproportionate increase in excitation lead to a more substantial increase in torque $(\sim 18 \%)$. However, many factors may affect the EMG-torque relationship that have not been taken into account. Those include, signal cancellation from overlapping positive and negative phases of action potentials (Day and Hulliger 2001); the sigmoid relationship between motor unit force and firing rate (Herbert and Gandevia 1999); and the nonlinear distribution of recruitment thresholds (Fuglevand et al. 1993). Interestingly, in a review, de Luca (1997) states that the amplitude of the 
EMG signal should be normalized to values less than $80 \%$ MVC. Above this level, the EMG signal is said to be exceptionally unstable and, hence, is unable to provide a suitable reference point. As shown in the present study, very large increases in EMG coincide with very small increases in calculated VA (Fig. 7a). This is consistent with unstable EMG measurements for similarly high torque levels.

EMG, superimposed torque and voluntary activation

When combining the non-linearity of the EMG-torque relationship and non-linearity of the superimposed triplettorque relationship, the EMG-torque relationship indicates that a large amount of extra EMG produces relatively little extra torque at high intensities. The triplet given to evoke the superimposed triplet could be considered as extra EMG in that three extra action potentials are added. As voluntary EMG is reduced in its effectiveness at producing torque in strong voluntary contractions, the extra activation from the triplet might be reduced similarly. This is in accordance with a model study of the adductor pollicis motoneuron pool (Herbert and Gandevia 1999). The authors predict large increases in motoneuronal excitation for minor increases in voluntary force and VA (as shown by small decreases in the superimposed twitch, their Fig. 9c). As mentioned above, this is consistent with the EMG-torque data in the present study (Fig. 7a). The small decreases in modelled interpolated twitch amplitude are also consistent with the current findings, where the triplet torque increment was only $3.2 \pm 1.1 \% \mathrm{MVC}$ at a torque level that corresponded to $79.6 \pm 6.1 \% \mathrm{MVC}$ and showed only a minor further reduction in size (to $1.0 \pm 0.9 \% \mathrm{MVC}$ ).

Note that the minor reduction in triplet increment with increasing voluntary torque is, and can only be, observed in those subjects consistently able to achieve high levels of VA, as would be expected from the model (Herbert and Gandevia 1999). This would indicate that these subjects are capable of excitation levels that (almost) evoke their MTC. Having noted this, it is important to point out that a $100 \%$ excitation may not be necessary to evoke the MTC as "'only' 60\% excitation was modelled to elicit $100 \%$ MTC (Herbert and Gandevia 1999). This is in agreement for example with the much greater excitation (EMG) levels attained during voluntary fast isometric knee extensions compared to during the plateau of an MVC (de Ruiter et al. 2004).

How can we be certain that our selected subjects did indeed approach their true MTC? The only way to truly determine the MTC of a muscle is by the use of tetanic nerve stimulation. In two subjects with exceptional ability for maximal VA (>99\%), supramaximal tetanic nerve stimulation $(2 \mathrm{~s}$, at $150 \mathrm{~Hz})$ of the knee extensors was performed and torque levels showed no further increase above MVC (data not presented). Applying supramaximal tetanic nerve stimulation is not recommended, however, as it is highly unpleasant and could be harmful to certain vulnerable subject groups (e.g. subjects with an ACL deficiency).

Currently, the ITT is the only feasible available technique used to determine VA of the knee extensors and, as mentioned previously, it is used extensively (Allen et al. 1995; Babault et al. 2003; Becker and Awiszus 2001; Behm et al. 1996, 2001; de Ruiter et al. 2004; McKenzie et al. 1992; Newman et al. 2003; Oskouei et al. 2003; Suter and Herzog 1997). The findings of the present study indicate that for the knee extensors, despite a commonly reported maximal VA of $\sim 90 \%$ for regular healthy subjects, a far greater relative torque increase seems possible than the $10 \%$ implied by $90 \%$ VA. This has important implications for conclusions that are drawn regarding calculated VA. Especially because many studies have used target force levels expressed as \%MVC to investigate: effects of caffeine neuromuscular function (Kalmar and Cafarelli 1999), the \%MVC at which full occlusion of the blood supply occurs (de Ruiter et al. 2006), and fatigue of the quadriceps in patients with multiple sclerosis for instance (de Haan et al. 2000). Clearly, knowledge of the potential of a far greater torque increase than, for example, $10 \%$ implied by a VA of $90 \%$ is very important.

In conclusion, when maximal VA is calculated to be $\sim 90 \%$ (as in regular healthy subjects), this probably represents a considerable overestimation of the subjects' ability to maximally drive their quadriceps muscles. Moreover, the effect that the length dependent size of the resting triplet has on the calculation of VA with the conventional method is only minimal when VA is greater than 95\%. Furthermore, a curvilinear shape of the EMG-force relationship may be caused by a disproportionately large increase in normalized rsEMG when MVC is approached in subjects with very high capacity to drive their muscles maximally.

\section{References}

Alkner BA, Tesch PA, Berg HE (2000) Quadriceps EMG/force relationship in knee extension and leg press. Med Sci Sports Exerc 32:459-463

Allen GM, Gandevia SC, McKenzie DK (1995) Reliability of measurements of muscle strength and voluntary activation using twitch interpolation. Muscle Nerve 18:593-600

Babault N, Pousson M, Ballay Y, Van Hoecke J (2001) Activation of human quadriceps femoris during isometric, concentric, and eccentric contractions. J Appl Physiol 91:2628-2634

Babault N, Pousson M, Michaut A, Van Hoecke J (2003) Effect of quadriceps femoris muscle length on neural activation during 
isometric and concentric contractions. J Appl Physiol 94:983990

Babault N, Desbrosses K, Fabre MS, Michaut A, Pousson M (2006) Neuromuscular fatigue development during maximal concentric and isometric knee extensions. J Appl Physiol 100:780-785

Becker R, Awiszus F (2001) Physiological alterations of maximal voluntary quadriceps activation by changes of knee joint angle. Muscle Nerve 24:667-672

Behm DG, St.-Pierre DM, Perez D (1996) Muscle inactivation: assessment of interpolated twitch technique. J Appl Physiol 81:2267-2273

Behm D, Power K, Drinkwater E (2001) Comparison of interpolation and central activation ratios as measures of muscle inactivation. Muscle Nerve 24:925-934

Belanger AY, McComas AJ (1981) Extent of motor unit activation during effort. J Appl Physiol 51:1131-1135

Bigland-Ritchie B, Furbush F, Woods JJ (1986) Fatigue of intermittent submaximal voluntary contractions: central and peripheral factors. J Appl Physiol 61:421-429

Bulow PM, Norregaard J, Danneskiold-Samsoe B, Mehlsen J (1993) Twitch interpolation technique in testing of maximal muscle strength: influence of potentiation, force level, stimulus intensity and preload. Eur J Appl Physiol Occup Physiol 67:462-466

Day S, Hulliger M (2001) Experimental simulation of cat electromyogram: evidence for algebraic summation of motor-unit action-potential trains. J Neurophysiol 86:2144-2158

Deutekom M, Beltman JG, de Ruiter CJ, de Koning JJ, de Haan A (2000) No acute effects of short-term creatine supplementation on muscle properties and sprint performance. Eur J Appl Physiol 82:223-229

Dowling JJ, Konert E, Ljucovic P, Andrews DM (1994) Are humans able to voluntarily elicit maximum muscle force? Neurosci Lett 179:25-28

Fuglevand AJ, Winter DA, Patla AE (1993) Models of recruitment and rate coding organization in motor-unit pools. J Neurophysiol 70:2470-2488

Gandevia SC, McKenzie DK (1988) Activation of human muscles at short muscle lengths during maximal static efforts. J Physiol 407:599-613

Grabljevec K, Burger H, Kersevan K, Valencic V, Marincek C (2005) Strength and endurance of knee extensors in subjects after paralytic poliomyelitis. Disabil Rehabil 27:791-799

de Haan A, de Ruiter CJ, van der Woude LHV, Jongen PJH (2000) Contractile properties and fatigue of quadriceps muscles in multiple sclerosis. Muscle Nerve 23:1534-1541

Herbert RD, Gandevia SC (1999) Twitch interpolation in human muscles: mechanisms and implications for measurement of voluntary activation. J Neurophysiol 82:2271-2283

Herbert RD, Gandevia SC, Allen GM (1997) Sensitivity of twitch interpolation. Muscle Nerve 20:521-523

Hisaeda HO, Shinohara M, Kouzaki M, Fukunaga T (2001) Effect of local blood circulation and absolute torque on muscle endurance at two different knee-joint angles in humans. Eur J Appl Physiol $86: 17-23$

Kalmar JM, Cafarelli E (1999) Effects of caffeine on neuromuscular function. J Appl Physiol 87:801-808

Kawakami Y, Akima H, Kubo K, Muraoka Y, Hasegawa H, Kouzaki M, Imai M, Suzuki Y, Gunji A, Kanehisa H, Fukunaga T (2001) Changes in muscle size, architecture, and neural activation after 20 days of bed rest with and without resistance exercise. Eur J Appl Physiol 84:7-12

Kooistra RD, de Ruiter CJ, de Haan A (2005) Muscle activation and blood flow do not explain the muscle length-dependent variation in quadriceps isometric endurance. J Appl Physiol 98:810-816
Kubo K, Tsunoda N, Kanehisa H, Fukunaga T (2004) Activation of agonist and antagonist muscles at different joint angles during maximal isometric efforts. Eur J Appl Physiol 91:349-352

de Luca CJ (1997) The use of surface electromyography in biomechanics. J Appl Biomech 13:135-163

Maffiuletti NA, Lepers R (2003) Quadriceps femoris torque and EMG activity in seated versus supine position. Med Sci Sports Exerc 35:1511-1516

McKenzie DK, Bigland-Ritchie B, Gorman RB, Gandevia SC (1992) Central and peripheral fatigue of human diaphragm and limb muscles assessed by twitch interpolation. J Physiol 454:643-656

Merton PA (1954) Voluntary strength and fatigue. J Physiol 123:553564

Muraoka T, Muramatsu T, Fukunaga T, Kanehisa H (2004) Influence of tendon slack on electromechanical delay in the human medial gastrocnemius in vivo. J Appl Physiol 96:540-544

Newman SA, Jones G, Newham DJ (2003) Quadriceps voluntary activation at different joint angles measured by two stimulation techniques. Eur J Appl Physiol 89:496-499

Ng AV, Agre JC, Hanson P, Harrington MS, Nagle FJ (1994) Influence of muscle length and force on endurance and pressor responses to isometric exercise. J Appl Physiol 76:2561-2569

Oskouei MA, Van Mazijk BC, Schuiling MH, Herzog W (2003) Variability in the interpolated twitch torque for maximal and submaximal voluntary contractions. J Appl Physiol 95:1648-1655

Pincivero DM, Coelho AJ (2000) Activation linearity and parallelism of the superficial quadriceps across the isometric intensity spectrum. Muscle Nerve 23:393-398

Place N, Maffiuletti NA, Ballay Y, Lepers R (2005) Twitch potentiation is greater after a fatiguing submaximal isometric contraction performed at short vs. long quadriceps muscle length. J Appl Physiol 98:429-436

Psek JA, Cafarelli E (1993) Behavior of coactive muscles during fatigue. J Appl Physiol 74:170-175

de Ruiter CJ, Jones DA, Sargeant AJ, de Haan A (1999) Temperature effect on the rates of isometric force development and relaxation in the fresh and fatigued human adductor pollicis muscle. Exp Physiol 84:1137-1150

de Ruiter CJ, Kooistra RD, Paalman MI, de Haan A (2004) Initial phase of maximal voluntary and electrically stimulated knee extension torque development at different knee angles. J Appl Physiol 97:1693-1701

de Ruiter CJ, de Boer MD, Spanjaard M, de Haan A (2005) Knee angle-dependent oxygen consumption during isometric contractions of the knee extensors determined with near-infrared spectroscopy. J Appl Physiol 99:579-586

de Ruiter CJ, Goudsmit JFA, van Tricht JA, de Haan A (2006) The isometric knee extension torque at which muscle reoxygenation stops in human quadriceps femoris muscle. Med Sci Sports Exerc (in press)

Rutherford OM, Jones DA, Newham DJ (1986) Clinical and experimental application of the percutaneous twitch superimposition technique for the study of human muscle activation. $\mathrm{J}$ Neurol Neurosurg Psychiat 49:1288-1291

Stephenson DG, Williams DA (1982) Effects of sarcomere length on the force-pCa relation in fast- and slow-twitch skinned muscle fibres from the rat. J Physiol 333:637-653

Suter E, Herzog W (1997) Extent of muscle inhibition as a function of knee angle. J Electromyogr Kinesiol 7:123-130

Suter E, Herzog W (2001) Effect of number of stimuli and timing of twitch application on variability in interpolated twitch torque. $\mathrm{J}$ Appl Physiol 90:1036-1040

Woods JJ, Bigland-Ritchie B (1983) Linear and non-linear surface EMG/force relationships in human muscles. An anatomical/ functional argument for the existence of both. Am J Phys Med 62:287-299 